\section{Le virobiote intestinal, nouvelle composante des interactions entre le microbiote et le système immunitaire}

Caroline Henrot, Maria Kuksin
École normale supérieure de Lyon, département de biologie, Master biologie, Lyon, France.
> Si les effets bénéfiques des bactéries du microbiote intestinal sur l'hôte ont été bien démontrés, ceux du microbiote viral, ou virobiote, commencent tout juste à être étudiés. La souris peut constituer un bon modèle pour l'étude fonctionnelle du virobiote. En effet, les virus entériques murins partagent des similarités avec les virus humains. En particulier, le norovirus murin (MNV, murine norovirus) est souvent retrouvé dans le virobiote intestinal de la souris. Les norovirus sont des virus intestinaux à ARN positif simple brin, de la famille des Caliciviridae, et sont la principale cause des gastro-entérites virales chez l'homme. Chez les souris immunocompétentes, le MNV n'est pas pathogène. Cependant, dans certaines lignées de souris portant une mutation dans un gène de susceptibilité aux maladies inflammatoires chroniques de l'intestin ( $\mathrm{MICl}$ ), ce virus commensal peut déclencher la maladie de Crohn [1]. Ce comportement ambivalent a déjà été décrit pour certaines bactéries du microbiote, associées au développement de $\mathrm{MICl}$ sous l'influence de facteurs génétiques [2]. Ces données posent la question de caractéristiques partagées entre le MNV et certaines bactéries du microbiote intestinal, notamment en termes d'effets sur le système immunitaire de l'hôte.

\section{L'infection par le MNV restaure la structure intestinale et l'activité immuno-physiologique des souris privées de microbiote bactérien} L'équipe de Ken Cadwell, de la New York University School of Medicine, s'est intéressée au rôle du MNV dans l'intestin en absence de microbiote bactérien
[3]. Pour cela, les chercheurs ont utilisé des souris axéniques et des souris traitées aux antibiotiques, qui présentent des anomalies dans la structure de la muqueuse intestinale, avec des villosités fines et cryptes étroites, ainsi que des différences de certaines populations de cellules immunitaires $(\rightarrow)$.

$(\rightarrow)$ Voir page 572 Ces souris ont été colonisées de façon artificielle par la souche MNV.CR6 : des couples parentaux ont été infectés oralement, permettant au virus de coloniser l'intestin et de se transmettre verticalement à la génération suivante, comme c'est le cas pour la transmission du microbiote bactérien. Étonnamment, l'architecture intestinale, ainsi que les populations de lymphocytes T et de cellules innées lymphoïdes (ILC pour innate lymphoid cells) de type 2 ont été restaurées par cette infection. Ainsi, un virus intestinal semble suffisant pour modifier l'architecture tissulaire et les populations immunitaires, indiquant que le virobiote pourrait avoir un effet bénéfique sur l'homéostasie intestinale, de façon similaire au microbiote bactérien. II est particulièrement intéressant de remarquer qu'ici, l'infection par une unique souche virale compense certaines anomalies causées par l'absence de très nombreuses espèces de bactéries.

Les auteurs ont alors réalisé des expériences de séquençage des ARN issus de cellules intestinales. Ils ont montré que le transcriptome des cellules intestinales est similaire pour les souris avec un microbiote bactérien et celles infectées par la souche virale MNV.CR6. Le même profil d'expression de gènes a été observé, en particulier pour des gènes régulant le développement du système immunitaire. Ceci corrobore l'idée que le virobiote et les bactéries commensales intestinales exercent des effets similaires sur le système immunitaire de l'hôte. Le MNV.CR6 induit également l'expression de gènes impliqués dans la réponse antivirale et en particulier dans la réponse interféron de type I (IFN-I), tels que IFIG-1. II faut de plus souligner que la compensation des anomalies intestinales induite par le MNV n'est pas observée chez des souris déficientes pour le récepteur des IFN-I (souris Ifnarl ${ }^{-/-}$), indiquant que les effets observés en présence du MNV.CR6 sont dépendants de cette réponse antivirale IFN-I.

\section{L'infection par le MNV protège des inflammations intestinales}

Suite à une inflammation intestinale induite chimiquement par l'équipe de Ken Cadwell, les souris traitées par des antibiotiques présentent un taux de survie plus faible que les souris témoins. Ainsi, la détérioration du microbiote intestinal bactérien, causée par le traitement antibiotique, rend les souris plus vulnérables à l'inflammation. Les auteurs ont observé que l'infection par le MNV.CR6 réduit les effets délétères du traitement antibiotique, indiquant que le MNV.CR6 pourrait avoir un rôle protecteur vis-à-vis de l'inflammation chez ces souris. En outre, chez des souris préalablement soumises à des antibiotiques, l'infection par MNV. CR6 diminue les conséquences de l'in- 


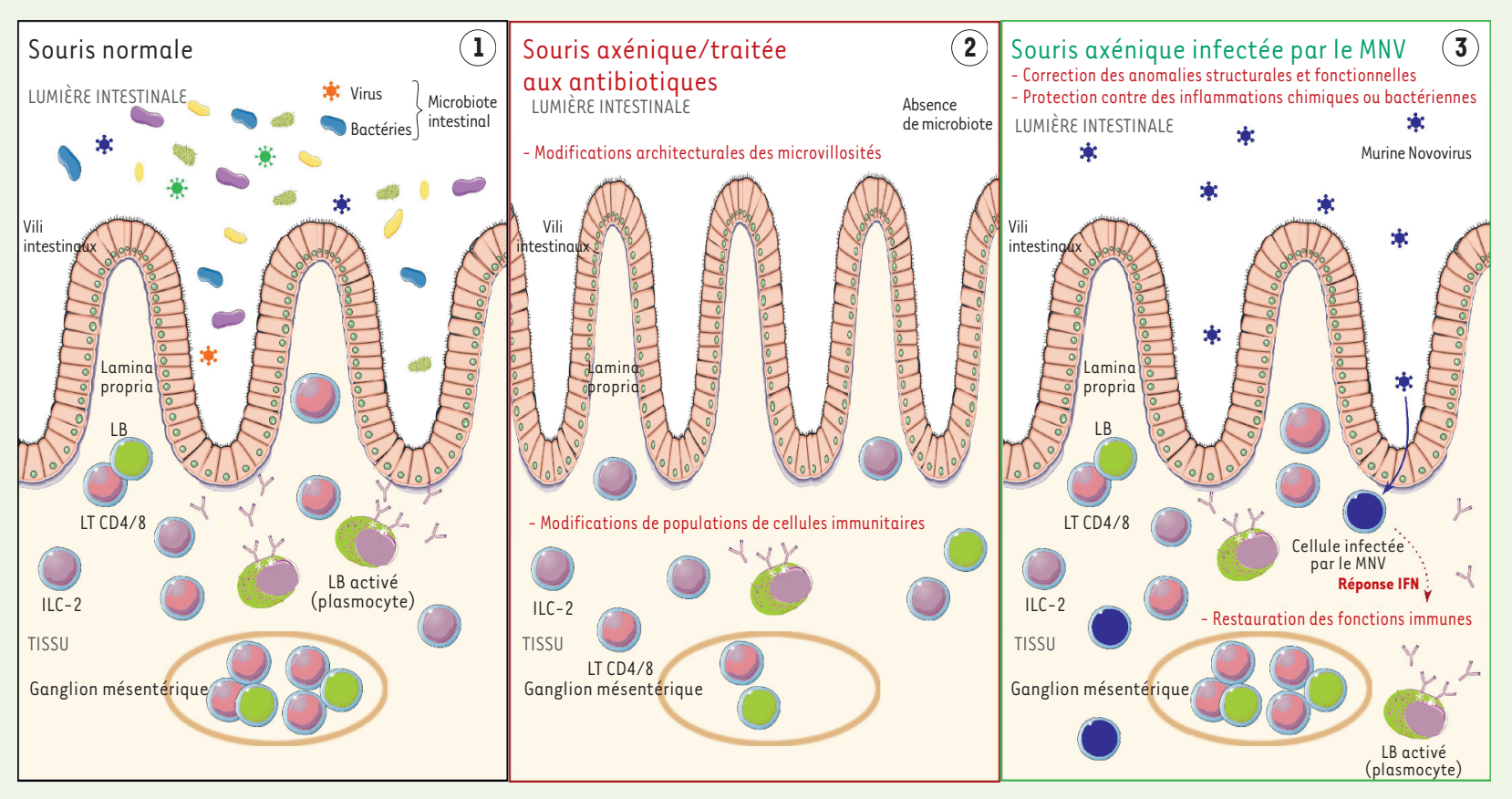

Figure 1. L'infection par le MNV corrige les anomalies intestinales des souris dépourvues de microbiote bactérien. 1. Intestin de souris saines, présentant un microbiote composé de bactéries et de virus. 2. Intestin de souris dépourvues de microbiote. On observe des vili anormalement fins, ainsi qu'une déplétion des lymphocytes T et un taux anormalement élevé de cellules lymphoïdes ILC-2, au niveau de la lamina propria et des ganglions mésentériques. Ces souris sont également plus vulnérables à l'inflammation. 3. L'infection par le MNV permet de restaurer la structure normale de la muqueuse ainsi que les fonctions immunes. La réponse antivirale de l'hôte (réponse interféron de type I, IFN), stimulée par le virus, serait un médiateur important de cet effet. L'infection par le MNV permet également de protéger les souris de l'inflammation induite par des agents chimiques ou bactériens.

fection par la bactérie entéropathogène Citrobacter rodentium, sans pour autant réduire la charge bactérienne. L’infection par MNV.CR6 réduit également la production de facteurs de virulence par $C$. rodentium, probablement en induisant la stimulation d'une réponse immunitaire antibactérienne. Ces résultats montrent qu'un virus peut avoir un effet protecteur contre des agents inflammatoires chimiques ou bactériens, chez des souris traitées par des antibiotiques et possédant une flore bactérienne diminuée.

Ainsi, ces travaux suggèrent que de façon similaire au microbiote bactérien, le virobiote intestinal pourrait stimuler le système immunitaire de l'hôte, lui permettant de mieux se défendre contre des agents infectieux ou noninfectieux (Figure 1). Le virobiote et le microbiote bactériens pourraient donc agir de façon étroitement liée et avoir des effets en partie redondants. Cela souligne la nécessité d'étudier plus en profondeur le rôle du virobiote sur la physiologie de l'hôte et ses interactions avec le microbiote bactérien.

\section{Vers la manipulation du virobiote en santé humaine?}

Ces observations ouvrent de nouvelles perspectives en santé humaine. La redondance entre les microbiotes bactérien et viral pourrait être particulièrement intéressante dans le contexte de la prise d'antibiotiques: les antibiotiques ont des effets délétères sur les populations bactériennes du microbiote, ce qui représente un effet secondaire néfaste important de ces traitements. Afin de restaurer la flore bactérienne, il est courant d'avoir alors recours à des probiotiques (des bactéries ou levures, aux effets bénéfiques pour la santé, notamment en cas de dysbiose) ou à des prébiotiques (des fibres alimentaires indigestes qui vont stimuler la croissance de certaines bactéries intestinales de types probiotiques). Certains virus pourraient-ils également permettre de compenser cette dysbiose bactérienne induite suite à un traitement antibiotique? II serait donc pertinent de déterminer s'il est possible de transposer à l'homme les résultats obtenus chez la souris avec le MNV. Cependant, l'étude du virome humain est très complexe, de par la grande diversité de ses populations virales, et du fait que la plupart des séquences virales trouvées dans le corps humain n'ont pas d'homologues dans les bases de données. Ce champ de recherche est en plein développement, grâce aux nouvelles techniques de séquençage et aux progrès de la bioinformatique pour l'analyse des métagénomes [4]. 
Par ailleurs, la transplantation de microbiote fécal est déjà utilisée en santé humaine pour soigner certaines inflammations intestinales dues à l'infection par Clostridium difficile et sont à l'étude pour d'autres pathologies [5]. Les travaux de Kernbauer et al. indiquent qu'il est possible qu'une partie des effets bénéfiques de la transplantation soit due aux virus transplantés, et pas seulement aux bactéries. À l'inverse, certains virus introduits au cours de la transplantation pourraient avoir des effets négatifs sur la physiologie des patients. Une meilleure compréhension de la composition du virobiote et de ses propriétés fonctionnelles est donc primordiale pour optimiser cette stratégie thérapeutique.

Une altération particulière du virome intestinal a récemment été identifiée dans le cas de cancers colorectaux $[6,7]$. Ces travaux suggèrent que le virobiote intestinal, et en particulier les populations de bactériophages, pourraient indirectement contribuer au développement de la pathologie, en modulant les populations bactériennes résidant dans l'intestin. L'analyse du virome pourrait donc être intéressante dans le diagnostic et le pronostic de de ces cancers. De la même manière que l'on parle de dysbiose bactérienne, on peut alors introduire la notion de «dysbiose virale », qui pourrait être un marqueur de certaines pathologies de la muqueuse intestinale [8]. Les effets du virobiote intestinal pourraient d'ailleurs s'élargir au-delà de l'intestin. En effet, une étude récente montre par exemple que des modifications de la composition du virome intestinal précédent le déclenchement du diabète de type I, dans une cohorte d'enfants susceptibles de développer la maladie [9]. L'ensemble de ces observations suggère donc que le virobiote intestinal pourrait contribuer à l'homéostasie de l'intestin et de l'organisme entier. La compréhension du virobiote reste donc à approfondir et pourrait permettre d'apporter des pistes pour l'élaboration de nouvelles stratégies préventives ou thérapeutiques en santé humaine. $\diamond$ The intestinal virobiota, a new component in the interactions between the microbiota and the immune system

\section{LIENS D'INTÉRÊT}

Les auteures déclarent n'avoir aucun lien d'intérêt concernant les données publiées dans cet article.

\section{RÉFÉRENCES}

1. Cadwell K, Patel KK, Maloney NS, et al. Virus-plussusceptibility gene interaction determines Crohn's disease gene Atg $16 \mathrm{Ll}$ phenotypes in intestine. Cell $2010 ; 141: 1135-45$.

2. Matsuoka K, Kanai T. The gut microbiota and inflammatory bowel disease. Semin Immunopatho $2015 ; 37: 47-55$.

3. Kernbauer $\varepsilon$, Ding Y, Cadwell K. An enteric virus can replace the beneficial function of commensal bacteria. Nature $2014 ; 16: 94-8$.

4. Abeles SR, Pride DT. Molecular bases and role of viruses in the human microbiome. J Mol Biol 2014 ; 426: 3892-906

5. Pamer $\varepsilon$ G. Fecal microbiota transplantation: effectiveness, complexities, and lingering concerns. Mucosal Immunol $2014 ; 7: 210-4$

6. Nakatsu G, Zhou H, Wu WKK, et al. Alterations in enteric virome are associated with colorectal cancer and survival outcomes. Gastroenterology 2018 ; 155 529-41.e5.

7. Hannigan GD, Duhaime MB, Ruffin MT, et al. Diagnostic potential and interactive dynamics of the colorectal cancer virome. MBio 2018 ; 9. pii: e0224818.

8. Cadwell K. The virome in host health and disease. Immunity 2015 ; 42 : 805-13.

9. Zhao G, Vatanen T, Droit L, et al. Intestinal virome changes precede autoimmunity in type I diabetessusceptible children. Proc Natl Acad Sci USA 2017 ; 114 : ع6166-75

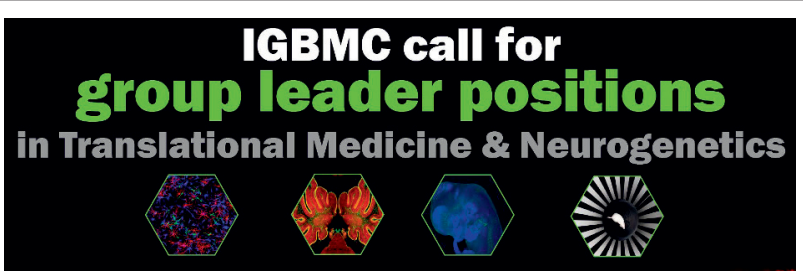

The Translational Medicine department (www.igbmc.fr) is welcoming applications for group leader positions (junior and senior) and aims to reinforce one of the following research axes:

- Neurological or muscle disorders with a focus on cellular biology, genetics, imaging, electrophysiology and/ or therapeutic development,

- Animal and cell (iPS, organoïds) models of neurological and/or muscle diseases,

- Bioinformatic and large-scale analysis dedicated to human pathologies.

Your application: Expression of interest will include, in a single PDF file, a curriculum vitae, a list of publications, names and contact of three referees, a brief description ( 2 to 4 pages) of past achievements and a detailed description (4 to 5 pages) of future research. Applications should be addressed to TMNcall2019@igbmc.fr subject: TMNcall2019.

The call is open until July $15^{\text {th }}$ 2019. Pre-selected candidates will be invited to the IGBMC to present a seminar and will be interviewed by the scientific direction of the IGBMC. 